\title{
A SEMIGROUP UNION OF DISJOINT LOCALLY FINITE SUBSEMIGROUPS WHICH IS NOT LOCALLY FINITE
}

\author{
T. C. Brown
}

The semigroup $S$ of the title is the free semigroup $F$ on four generators factored by the congruence generated by the set of relations $\left\{w^{2}=w^{3} \mid w \in F\right\}$. The following lemma is proved by examining the elements of a given congruence class of $F$ :

Lemma. If $x, y \in S$ and $x^{2}=y^{2}$, then either $x y=x^{2}$ or $y x=x^{2}$.

From the Lemma it then easily follows that the (disjoint) subsemigroups $\left\{y \in S \mid y^{2}=x^{2}\right\}$ of $S$ are locally finite.

This note answers in the negative a question raised by Shevrin in [2].

THEOREM. There exists a semigroup $S$ with disjoint locally finite subsemigroups $S_{e}$ such that $S=\cup S_{e}$ and $S$ is not locally finite.

Let $F$ be the free semigroup with identity on four generators. Let $\sim$ denote the smallest congruence on $F$ containing the set $\left\{\left(x^{2}, x^{3}\right) \mid x \in F\right\}$. That is, for $w, w^{\prime} \in F, w \sim w^{\prime}$ if and only if a finite sequence of "transitions", of either of the types $a b^{2} c \rightarrow a b^{3} c$ or $a b^{3} c \rightarrow$ $a b^{2} c$, transforms $w$ into $w^{\prime}$.

The equivalence classes of $F$ with respect to $\sim$ are taken as the elements of $S$, and multiplication in $S$ is defined in the natural way.

There is given in [1] a sequence on four symbols in which no block of length $k$ is immediately repeated, for any $k$. Thus the left initial segments of this sequence give elements of $F$ containing no squares. Since no transition of the form $a b^{2} c \rightarrow a b^{3} c$ or $a b^{3} c \rightarrow a b^{2} c$ can be applied to an element of $F$ containing no squares, the equivalence classes containing these elements consist of precisely one element each; thus the semigroup $S$ is infinite, and hence not locally finite.

In what follows, the symbols $\alpha, \alpha_{1}, \alpha_{2}, \cdots$ refer to transformations (on elements of $F$ ) of the form

$$
a b \rightarrow a y b, \text { where } a \sim a y \text {, and } a, b, y \in F .
$$

The symbols $\beta, \beta_{1}, \beta_{2}, \cdots$ refer to transformations of the type

$$
a x b \rightarrow a b, \text { where } a \sim a x \text {, and } a, b, x \in F .
$$

Note that $a b^{2} c \rightarrow a b^{3} c$ is an $\alpha$, and $a b^{3} c \rightarrow a b^{2} c$ in a $\beta$. 
LEMMA 1. If $w, w^{\prime} \in F$, and $w \beta \alpha=w^{\prime}$, then there are $\alpha_{1}, \beta_{1}$ such that $w \alpha_{1} \beta_{1}=w^{\prime}$.

Proof. Let

$$
w=a x b, \quad w \beta=a b, \text { where } a \sim a x .
$$

Let

$$
w \beta=A B, \quad w \beta \alpha=A y B, \text { where } A \sim A y .
$$

There are two cases:

(i) $A$ is contained in $\alpha$. That is,

$$
a=A a^{\prime} \text { and } w^{\prime}=w b \alpha=a \beta \alpha=A a^{\prime} b \alpha=A y a^{\prime} b .
$$

Here let

$$
w \alpha_{1}=a x b \alpha_{1}=A a^{\prime} x b \alpha_{1}=A y \alpha^{\prime} x b .
$$

Now since

$$
A y a^{\prime} \sim A a^{\prime}=a \sim a x=A a^{\prime} x \sim A y a^{\prime} x,
$$

we may let

$$
w \alpha_{1} \beta_{1}=A y a^{\prime} x b \beta_{1}=A y a^{\prime} b=w^{\prime} .
$$

(ii) $A$ is not contained in $a$. That is,

$$
b=b_{1} b_{2}, \quad A=a b_{1}, \quad A \sim A y,
$$

and

$$
w^{\prime}=w \beta \alpha=a b \alpha=a b_{1} b_{2} \alpha=A b_{2} \alpha=A y b_{2}=a b_{1} y b_{2} .
$$

Since

$$
a x b_{1} \sim a b_{1}=A \sim A y=a b_{1} y \sim a x b_{1} y,
$$

we may let

$$
w \alpha_{1}=a x b \alpha_{1}=a x b_{1} b_{2} \alpha_{1}=a x b_{1} y b_{2},
$$

and

$$
w \alpha_{1} \beta_{1}=a x b_{1} y b_{2} \beta_{1}=a b_{1} y b_{2}=w^{\prime}
$$

LEMMA 2. If $w, w^{\prime} \in F, w \gamma_{1} \gamma_{2} \cdots \gamma_{m}=w^{\prime}$, where each $\gamma_{i}$ is either an $\alpha$ or a $\beta$, then there are $\alpha_{1}, \cdots, \alpha_{n}, \beta_{1}, \cdots, \beta_{k}$ such that $w \alpha_{1} \cdots$ $\alpha_{n} \beta_{1} \cdots \beta_{k}=w^{\prime}$.

This follows immediately from Lemma 1 by induction. 
Lemma 3. The word abo contains a left initial segment which is equivalent to $a$.

Proof. Let $a b=A B, a b \alpha=A y B$, where $A \sim A y$. Again there are two cases:

(i) $A$ is contained in $a$. That is, $a=A a^{\prime}, a b \alpha=A a^{\prime} b \alpha=A y a^{\prime} b$. Since $A \sim A y$, the left initial segment $A y \alpha^{\prime}$ of $a b \alpha$ is equivalent to $a$.

(ii) $A$ is not contained in $a$. That is, $b=b_{1} b_{2}, A=a b_{1}, a b \alpha=$ $a b_{1} b_{2} \alpha=a b_{1} y b_{2}$.

Here, $a$ itself is a left initial segment of $a b \alpha$, and is certainly equivalent to $a$.

Lemma 4. If $x, y \in F$ and $x^{2} \sim y^{2}$, then either $y \sim x a$ for some $a \in F$, or $x \sim y b$ for some $b \in F$.

Proof. By Lemma 2, there are $\alpha_{i}$ and $\beta_{j}$ such that $x x \alpha_{1} \ldots$ $\alpha_{m} \beta_{1} \cdots \beta_{n}=y y$. Let $w=x x \alpha_{1} \cdots \alpha_{m}=y y \beta_{n}^{-1} \cdots \beta_{1}^{-1}$. By Lemma 3, $w$ contains a left initial segment $A$ equivalent to $x$. Similarly, since each $\beta_{i}^{-1}$ is an $\alpha, w$ also contains a left initial segment $B$ equivalent to $y$. Depending on which segment contains the other, either $B=A a$ for some $a$, or $A=B b$ for some $b$. In the first case, $y \sim B=A a \sim x a$; in the second, $x \sim A=B b \sim y b$.

Lemma 5. In this lemma, "=" will denote equality in S. Let $e$ be an idempotent element of $S: e=e^{2}$. Let $S_{e}=\left\{x \in S \mid x^{2}=e\right\}$. Then $S_{e}$ is a locally finite subsemigroup of $S$.

Proof. First, we note that $z \in S_{e}$ implies $e z=z e=e$. For $e z=$ $z^{2} z=z^{2}=e$, and similarly $z e=e$. Now let $x, y \in S_{e}$, that is, $x^{2}=$ $y^{2}=e$. By Lemma 4, either $y=x a$ or $x=y b$. In the first case, we obtain $x y=x^{2} a=x^{3} a=x^{2} y=e y=e$. In the second case, we obtain similarly that $y x=e$. Thus $x, y \in S_{e}$ implies $x y=e$ or $y x=e$. In either case, $(x y)^{2}=e$, that is, $x y \in S_{e}$. Thus $S_{e}$ is a semigroup.

Now let $x_{1}, \cdots, x_{n} \in S_{e}$, and let $\left\langle x_{1}, \cdots, x_{n}\right\rangle$ denote the subsemigroup of $S_{e}$ generated by $x_{1}, \cdots, x_{n}$. If $n=1$, then $\left\langle x_{1}\right\rangle$ is clearly finite; so suppose $n>1$. Then every element of $\left\langle x_{1}, \cdots, x_{n}\right\rangle$ may be expressed as a product of not more than $n$ of the $x_{i}$ 's. For any product $z$ of more than $n x_{i}$ 's must contain some $x_{i}$ twice: $z=a x_{i} b x_{i} c$, where $a, b, c \in S_{e}$. Since either $x_{\imath} b=e$ or $b x_{i}=e$, it follows that $x_{i} b x_{\imath}=e$ and $z=a e c=e c=e=x_{1} x_{1}$. This shows that $\left\langle x_{1}, \cdots, x_{n}\right\rangle$ is finite, and hence that $S_{e}$ is locally finite. 
The theorem follows immediately from Lemma 5, since clearly $e \neq e^{\prime}$ implies that $S_{e}$ and $S_{e^{\prime}}$ are disjoint, and

$$
S=\cup S_{e} \text {. }
$$

\section{REFERENCES}

1. R. A. Dean, A sequence without repeats on $x, x^{-1}, y, y^{-1}$, Amer. Math. Monthly 72 (1965), 383-385 (Math. Rev. 31, No. 3500).

2. L. N. Shevrin, On locally finite semigroups, Doklady Akad. Nauk SSSR 162 (1965), 770-773 = Soviet Math. Dokl. 6 (1965), 769.

Received December 19, 1966. Partially supported by Canadian NRC grant no. A-3983.

Simon Fraser University 